\title{
Generation of lossy mode resonances in planar waveguides towards development of humidity sensors
}

\author{
Omar Fuentes, Jesus M. Corres, Member, IEEE, Ignacio R. Matias, Senior Member, IEEE, Ignacio \\ Del Villar
}

\begin{abstract}
Lossy mode resonances (LMRs) are typically obtained with optical fibre. The Kretschmann configuration is an alternative but LMRs are generated with angles approaching grazing incidence. In this work, a new setup is explored, based on the lateral incidence of light on conventional planar waveguides such as glass slides or coverslips. Indium tin oxide was deposited onto both types of waveguides generating LMRs. The results of the simulations carried out agree well with the experimental results. As an example of the potential of this new and simple optical configuration, a humidity sensor with a sensitivity of $0.212 \mathrm{~nm} / \% \mathrm{RH}$ in the range from 65 to $90 \%$ of $\mathrm{RH}$ was developed, which expedites the development of other types of sensors already explored with LMR based optical fibre sensors.
\end{abstract}

Index Terms - Thin-films, Lossy Mode Resonance, Sensors, Transparent conductive coatings, Humidity sensors.

\section{INTRODUCTION}

$I^{n}$ recent decades, progress has been achieved in the domain of sensors thanks to the ability to deposit thin-films. One of the major milestones was achieved in 1982 [1] with the development of the first surface plasmon resonance sensor (SPR). The sensor was based on the utilization of the Kretschmann-Raether configuration [2], which consists of an optical prism on which a metallic thin-film coating is deposited. By introducing light with different angles of incidence, a surface plasmon polariton is excited in the metaldielectric interface at a specific angle range, something that is also observed as a function of wavelength.

Another phenomenon, lossy mode resonance (LMR) [3-4], can be observed with the same configuration [5]. That is why LMRs have been sometimes confused in the literature [3]. However, LMR generation requires a thin-film with different properties than those suitable for SPR generation. SPRs are

Manuscript received on $\mathrm{xx}, 2018$. This work was supported by the Spanish Agencia Estatal de Investigación (AEI) and Fondo Europeo de Desarrollo Regional (FEDER) (TEC2016-78047-R) (TEC2016-79367-C2-2-R) and by Pinar del Río University.

Omar Fuentes is with the Department of Telecommunications and Electronics of Pinar del Río University, Cuba (e-mail: omarf@upr.edu.cu).

Ignacio Del Villar, Jesus M. Corres and Ignacio R. Matias are with the Institute of Smart Cities and the Electrical and Electronic Engineering Department of the Public University of Navarre, 31006 Pamplona, Navarre, Spain (e-mails: imcorres@unavarra.es, natxo@unavarra.es, ignacio.delvillar@unavarra.es)

Copyright (C) 2018 IEEE obtained when the real part of the thin-film permittivity is negative and higher in magnitude than both its own imaginary part and the permittivity of the material surrounding the thinfilm, whereas LMRs occur when the real part of the thin-film permittivity is positive and higher in magnitude than both its own imaginary part and the material surrounding the thin-film $[3,6]$. In view of the previous conditions, one would think that it is not possible to simultaneously observe both phenomena, SPR and LMR. However, there are materials, such as indium tin oxide (ITO), that present different properties depending on the operating wavelength range due to the material dispersion. Thanks to this property, it has been possible to obtain both an SPR and an LMR with the Kretschmann-Raether configuration and to compare their properties [5,7].

Some important differences between SPRs and LMRs were observed. LMRs can be excited with both transverse electric (TE) and transverse magnetic (TM) polarized light, whereas SPRs can only be obtained at TM polarization [3]. The second difference is that, unlike SPR, the position of LMRs in the optical spectrum depends directly on the coating thickness, which allows for simple tuning of the resonance wavelength [8]. Finally, the range of angles of incidence for excitation of LMRs is quite different from those suitable for SPR generation. SPRs are typically obtained for angles ranging between $40^{\circ}$ and $70^{\circ}$ [9], whereas LMRs typically arise at near-grazing angle incidence, i.e. angles approaching $90^{\circ}$ [5]. This explains why most of the experimental work on LMRs uses optical fibre instead of the Kretschmann-Raether configuration [7, 10-15]. With this last configuration, it is very difficult to impinge light at nearly $90^{\circ}$. However, though optical fibres show good characteristics such as small size, immunity to electromagnetic interference (EMI), multiplexing capacity, a wide range of operating temperatures, and remote sensing capacity, due to their short diameter, they are easily breakable so there is a need to splice the sensor head, and they are affected by curvatures.

In this work, a planar waveguide was used as an alternative for optical fibre and the Kretschmann-Raether configuration. The setup consisted of the incidence of light by one of the lateral sides of a planar waveguide. For the sake of comparison, two cases were analyzed: a standard soda lime glass slide and a coverslip, which is thinner than the glass slide. The material selected for the nanocoating was ITO, a widely explored material in LMR-based sensors [7, 10], which 
allows comparison of its performance. Regarding the wavelength range under analysis, the VIS/NIR range (i.e. 400 to $1000 \mathrm{~nm}$ ) was selected because light sources and detectors are available at a relatively low cost.

The results presented in this work show that LMRs can be generated with both substrates (standard soda lime glass slide and coverslip), though their performance is slightly different.

Finally, as an example of the practical applicability of this novel optical configuration, the detection of relative humidity (RH) is explored. The knowledge of this parameter is important in many chemical, physical or biological processes, becoming critical in the final quality of biotechnology, pharmaceutical or food products, as well as in places where it is necessary to preserve health and comfort $[16,17,18]$.

\section{METHODS AND MATERIALS}

The experimental setup is described in Fig. 1. Light from an ASBN-W tungsten-halogen broadband source from Spectral Products Inc. (Putnam, FL, USA) was launched into a multimode optical fibre from Ocean Optics $(200 / 225 \mu \mathrm{m}$ of core/cladding diameter). This fibre was placed in front of one of the lateral sides of a planar waveguide and the output light was collected by another multimode optical fibre whose end was connected to an HR4000 spectrometer (OceanOptics Inc., Largo, FL, USA). As planar waveguides, RS France microscope slides $(75 \times 25 \times 1.1 \mathrm{~mm})$ and coverslips $(18 \times 18 \times 0.15 \mathrm{~mm})$, both of them made of soda lime glass, were used [19]. The planar waveguide was placed on a poly(methyl methacrylate) (PMMA) substrate material (Fig. 1), which allowed supporting the waveguide and aligning the fibres correctly on both sides of it. The thickness of the PMMA substrate was $5 \mathrm{~mm}$ and it presented a lower refractive index than the soda lime waveguides [20].

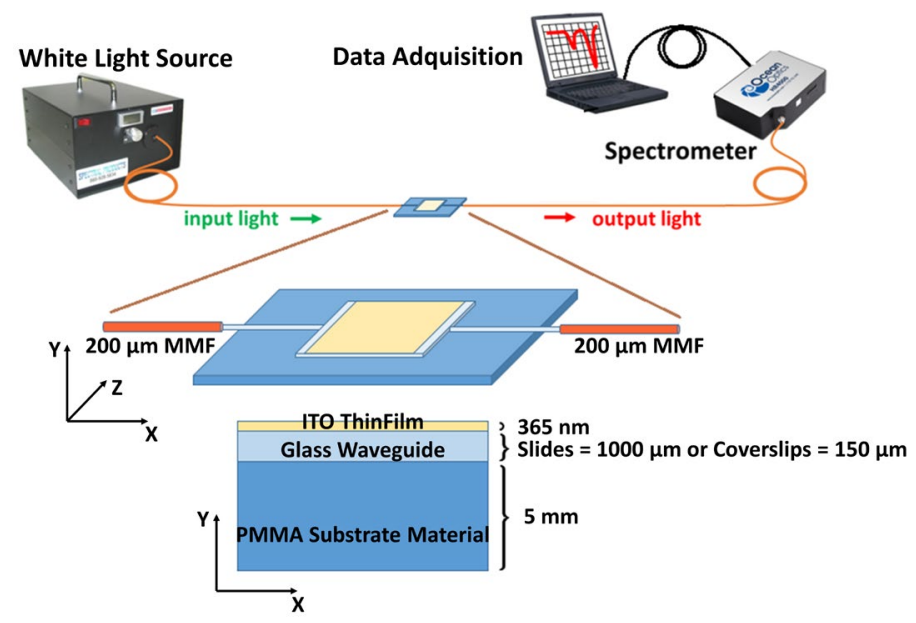

Fig. 1. Experimental setup

The setup was introduced in a DC sputtering machine (K675XD from Quorum Technologies, Ltd.) in order to deposit an indium tin oxide (ITO) thin-film on the waveguide. The ITO target used for the deposition presented $99.99 \%$ purity and it was purchased from ZhongNuo Advanced Material Technology Co. The parameters used for the deposition were partial pressure of argon $8 \times 10^{-2}$ mbar and intensity $150 \mathrm{~mA}$. The glass waveguide was positioned at a distance of $7 \mathrm{~cm}$ from the target and the optical spectra were monitored continuously during the deposition process.

A microscope image of the ITO deposition is shown in Fig. 2, taken with a field emission scanning electron microscope (SEM) UltraPlus, from CarlZeiss Inc., with an in-lens detector at $3 \mathrm{kV}$ and an aperture diameter of $30 \mu \mathrm{m}$.

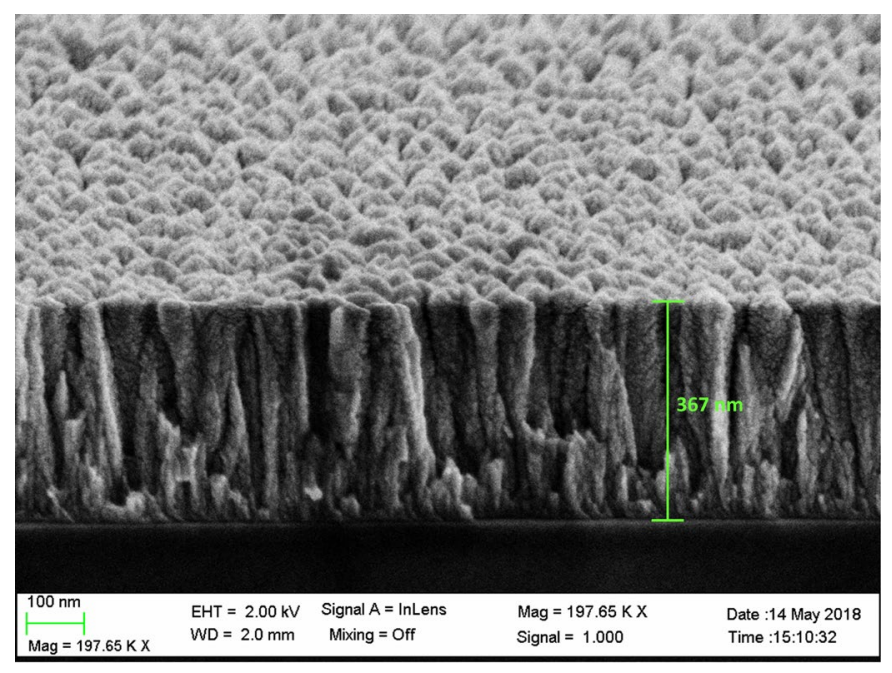

Fig. 2. Image obtained with the SEM microscope showing the ITO deposition thickness.

The refractive index and extinction coefficient of the ITO thin-film was also characterized with an ellipsometer UVISEL, with a spectral range of $0.6-6.5 \mathrm{eV}(190-2100 \mathrm{~nm})$, an angle of incidence of $70^{\circ}$, a spot size of $1 \mathrm{~mm}$, and software DeltaPsi2TM (from Horiba Scientific Thin Film Division). The estimated thickness of the coating was $362.5 \mathrm{~nm}$, which agrees well with the value obtained in Fig. 2 with the SEM microscope, whereas the refractive index is represented in Fig. 3.

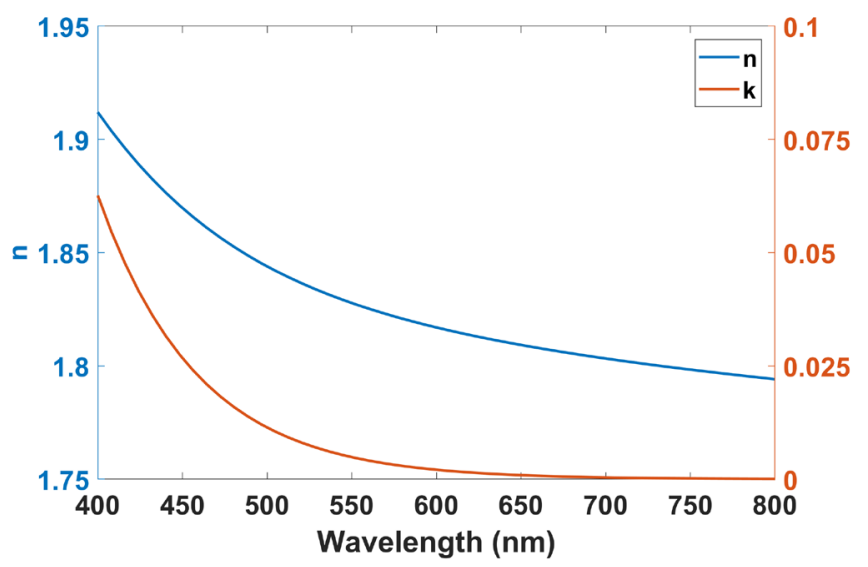

Fig. 3. Refractive index (n) and extinction coefficient (k) of ITO thin-film.

\section{NUMERICAL ANALYSIS}

With the parameters reported in section II a numerical 
analysis was performed of the transmission optical spectrum as a function of thickness for the coverslip waveguide. In order to analyze this structure FIMMWAVE® was used. The propagation was obtained with FIMMPROP, a module integrated with FIMMWAVE. The finite difference method (FDM) with the Quasi 2D version was used because the structure shows no variation in the $\mathrm{x}$ axis. In addition to this, 50 modes were analyzed to achieve a good convergence. Regarding the source, a Gaussian profile with full width at half maximum of $200 \mu \mathrm{m}$ was applied. It must be noted that the thin-film thickness plays an important role in the properties of indium tin oxide thin film, especially in the conductivity [21]. That is why the gradient of the imaginary part of the refractive index was taken into account in the simulation according to this expression that best fitted the experimental results:

$$
k=0.012 t^{2} \exp (-0.017 \lambda)
$$

where $t$ is the thin-film thickness and $\lambda$ is the wavelength in nm.

The results obtained in Fig. 4 show, as a function of the coating thickness, the average transmission value between the spectrum obtained for TE polarization and the spectrum obtained for TM polarization. The first LMR is clearly subdivided into two resonances: $\mathrm{LMR}_{\mathrm{TE}}$ and $\mathrm{LMR}_{\mathrm{TM}}$, whereas for the second LMR these two resonances, corresponding to both polarizations, overlap each other.

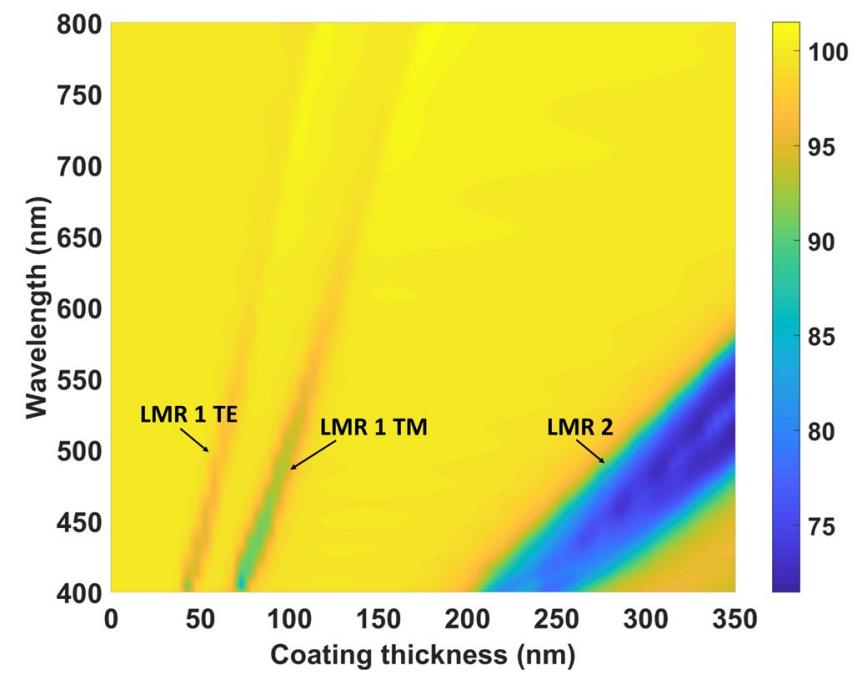

Fig. 4. Spectral response of the optical device as a function of coating thickness. Theoretical simulation

This effect can also be observed in Fig. 5, where the spectra of the second $\mathrm{LMR}_{\mathrm{TE}}$, the second $\mathrm{LMR}_{\mathrm{TM}}$ and the transmission average value between both of them is shown, proving the overlap between both polarizations [3]. Comparing the first and the second LMR, it is also evident that the depth of the resonance for the first LMR is very small compared to the second LMR. Moreover, at longer wavelengths the resonances are nearly imperceptible.

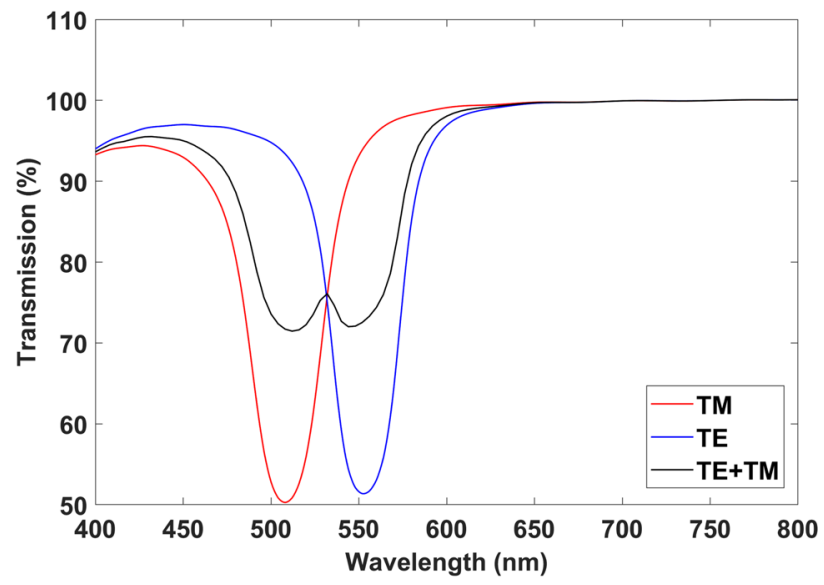

Fig. 5: Theoretical simulation of the optical spectra of the second LMR for TE and TM polarization together with the average between both of them, TE and TM. The thickness of the ITO thin-film is $350 \mathrm{~nm}$.

\section{LOSSY MOdE RESONANCE GENERATION}

During the deposition process, as explained in section II, the optical spectrum was continuously monitored using two different planar waveguides: a coverslip and a glass slide. The coverslip (see Fig. 6a), which was the one simulated in section III, shows the TE and TM resonances of the first LMR and the second resonance where both polarizations overlap each other. This agrees well with the simulation results of Fig. 4.

Regarding the glass slide (Fig. 6b), the resonances are generated after similar deposition times to those observed with the coveslip: the TE and TM resonances of the first LMR are visible after 2 and 4 minutes of deposition and the second LMR, where the TE and the TM resonance overlap each other, is visible after 14 minutes.

This non-dependence on the waveguide thickness in terms of generation of the LMRs with a progressive deposition of a thin-film has been previously observed with optical fiber waveguides [22]. The LMRs are generated after the same deposition time for different diameters of the optical fiber. This behavior is attributed to the fact that the optical waveguide is much thicker than the thin-film. Consequently, it can be assumed that the thin-film is surrounded by two infinite media (i.e. the optical waveguide and the outer medium). In fact, the application of optical models where these two media are considered infinite has permitted to obtain a perfect agreement between the experimental and the theoretical results $[8,10]$.

However, by comparing the results obtained with the converslip and the slide in Fig. 6, an important difference can be found in terms of signal intensity. The intensity for the resonances in the glass slide is lower because the waveguide is thicker and, consequently, the evanescent field is lower, whereas the opposite is true for the coverslip. This idea again agrees well with what was observed with optical fibre, where tapered optical fibres with reduced diameter presented deeper resonances than standard multimode fibre [22]. 

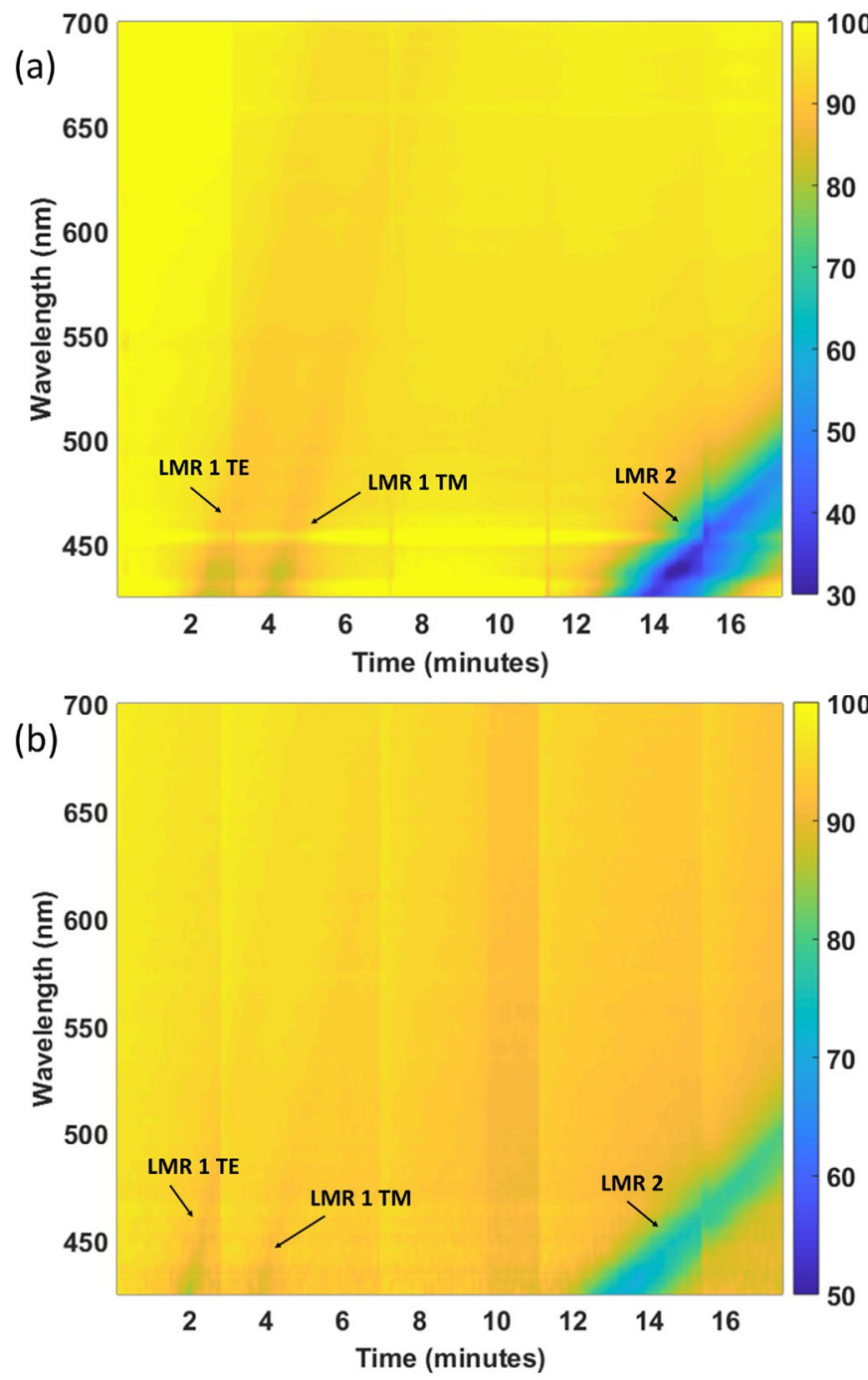

Fig. 6. Spectral response of the optical device as a function of deposition time: (a) coverslip; (b) glass slide.

According to the design rules of LMR-based sensors, the highest sensitivity is obtained with the first LMR $[3,8]$. However, as it was observed in the simulation, the first LMR with an ITO coating is almost undetectable, because it disappears as the deposition is being built up and the LMR moves to longer wavelengths (Fig. 6a and 6b). This is the reason for using the second LMR as a humidity sensor, even though it has a lower sensitivity $[3,8]$.

The difference in resonance intensity, observed when comparing the results obtained with a glass slide and with a coverslip in Fig. 6, is confirmed by analyzing the spectra after the deposition process (Fig. 7). It can be observed that the coverslip has a greater amplitude of the peak with respect to the slides. At a wavelength of $500 \mathrm{~nm}$, the coverslip has an attenuation of $40 \%$, whereas the glass slide has $12 \%$ attenuation.
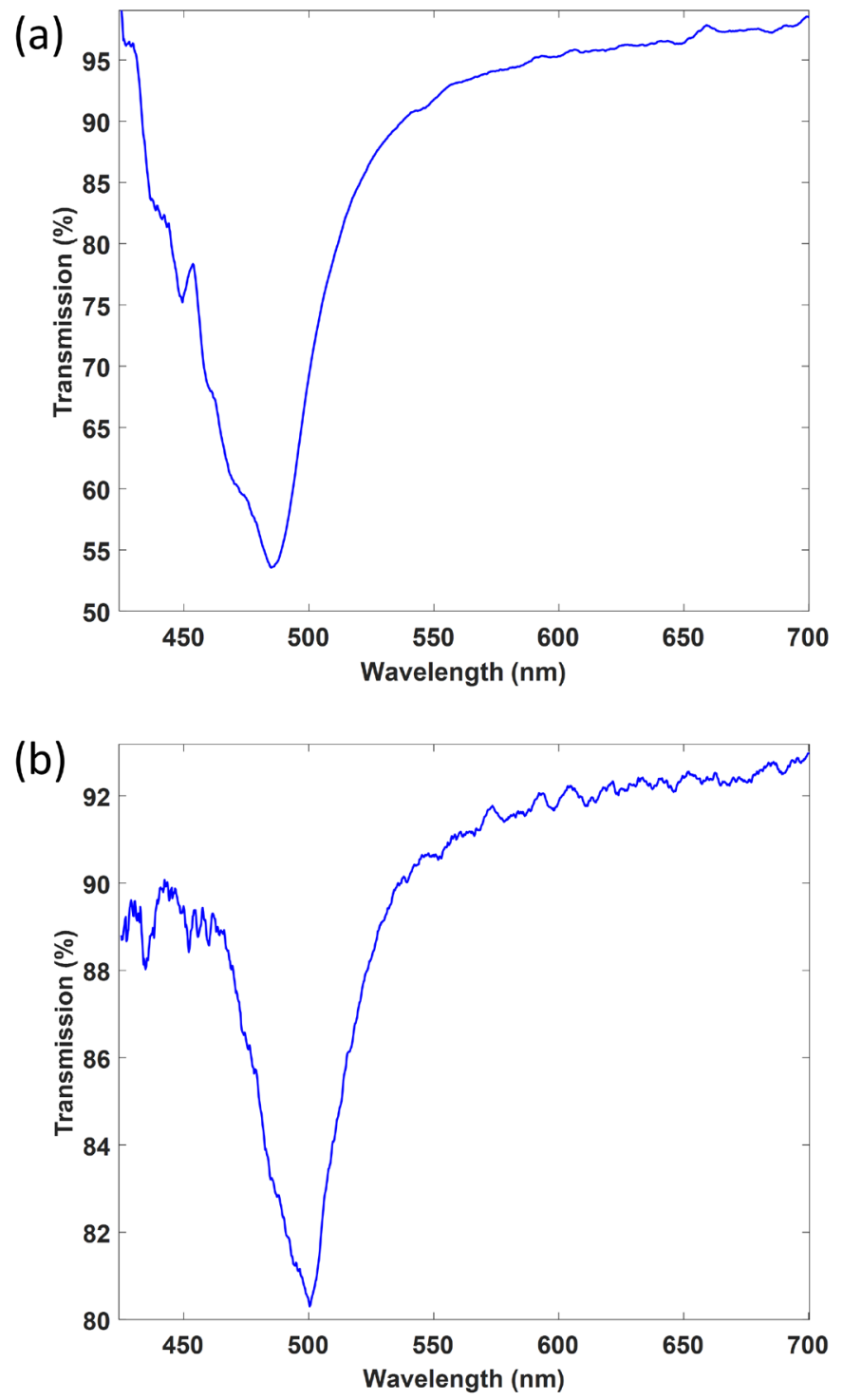

Fig. 7. Optical spectrum after 16 minute deposition of ITO with the sputtering machine: (a) coverslip; (b) glass slide. The second LMR is obtained at approximately the same wavelength and it is deeper in the coverslip case.

\section{Characterization OF THE Relative Humidity (RH) SENSOR}

For the characterization of the waveguides, a climate chamber type Binder KMF-115 series was used. Two types of tests were carried out, one based on ramps of 30 to $90 \%$ and 90 to $30 \%$ RH (Fig. 8) and the other one on steps of $10 \%$, in the same range (Fig. 9).

In order to avoid signal drifts, a thermal treatment in the presence of a high relative humidity was applied before characterizing the sensor, by following a method similar to that used in [23]. The parameters were $85 \% \mathrm{RH}$ and a temperature $50{ }^{\circ} \mathrm{C}$ for 16 hours.

The results show a wavelength shift of $7 \mathrm{~nm}$ (sensitivity 0.116 $\mathrm{nm} / \%$ of $\mathrm{RH}$ ) for the coverslip, whereas for the glass slide the wavelength shift is $5 \mathrm{~nm}$ (sensitivity $0.083 \mathrm{~nm} / \%$ of RH). 
In addition, the coverslip allows better tracking of the evolution of the signal thanks to the deeper resonance. This effect is more evident in Fig. 9, where the humidity is modified in steps and the response to the presence of noise is better.

If we compare these results with LMR based optical fiber sensors deposited with ITO (sensitivity $0.283 \mathrm{~nm} / \%$ of RH), the sensitivity is lower but it must be pointed out that here we are working with the second LMR, which typically presents a lower sensitivity [24].

(a)
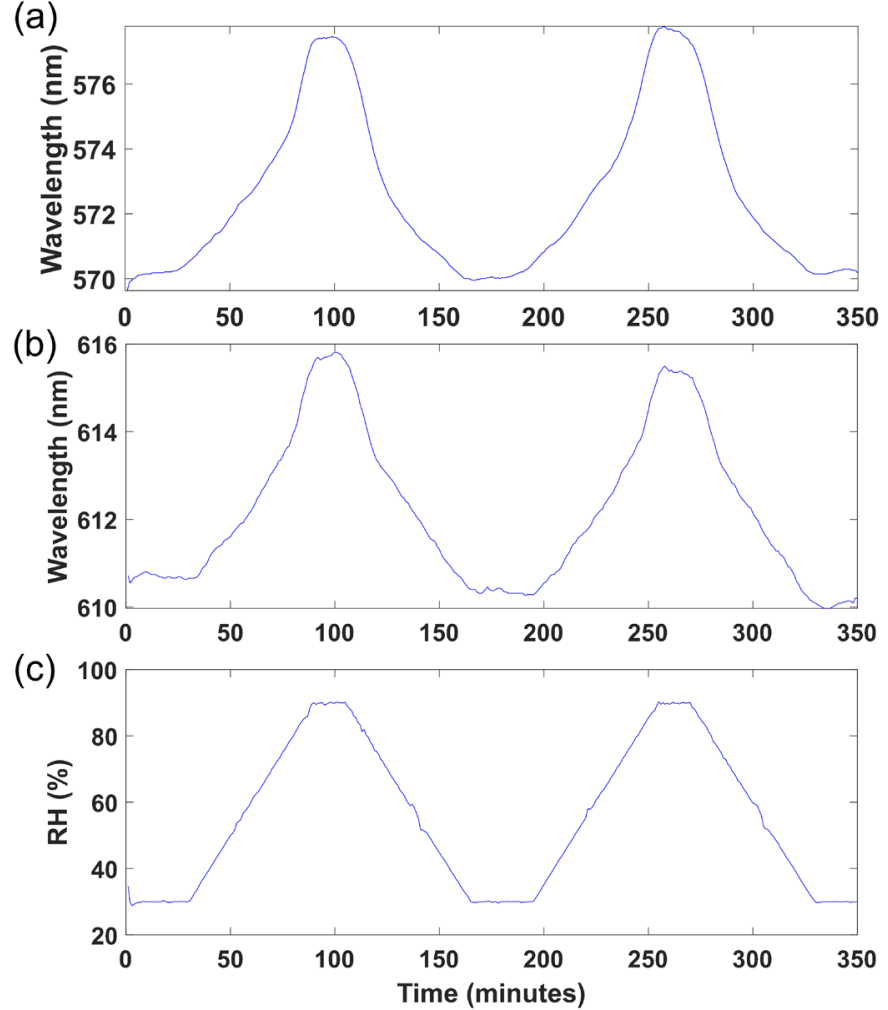

Fig. 8. Wavelength shift as a function of the RH in ramps from 30 to $90 \%$ : (a) coverslip; (b) slide. The relative humidity measured by the electronic sensor is shown in (c).

It is also observed that the response of the two sensors is similar and non-linear, being more sensitive at high relative humidity compared to low relative humidity. In both cases the relative humidity range can be divided into two regions (30 to $65 \%$ and 65 to $90 \%$ ). The RH in the range of $65 \%$ to $90 \%$ shows a sensitivity of $0.212 \mathrm{~nm} / \%$, whereas in the range $30 \%$ to $65 \%$ a sensitivity of $0.0657 \mathrm{~nm} / \%$ of $\mathrm{RH}$ is obtained.

In Fig. 10 the cross-sensitivity to temperature is analyzed in a range from $20^{\circ} \mathrm{C}$ to $50^{\circ} \mathrm{C}$ for the ITO coated glass slide. Some fluctuations are observed while introducing temperature changes but on average the effect of temperature in this range analyzed is negligible compared to the wavelength shift observed as a function of humidity.
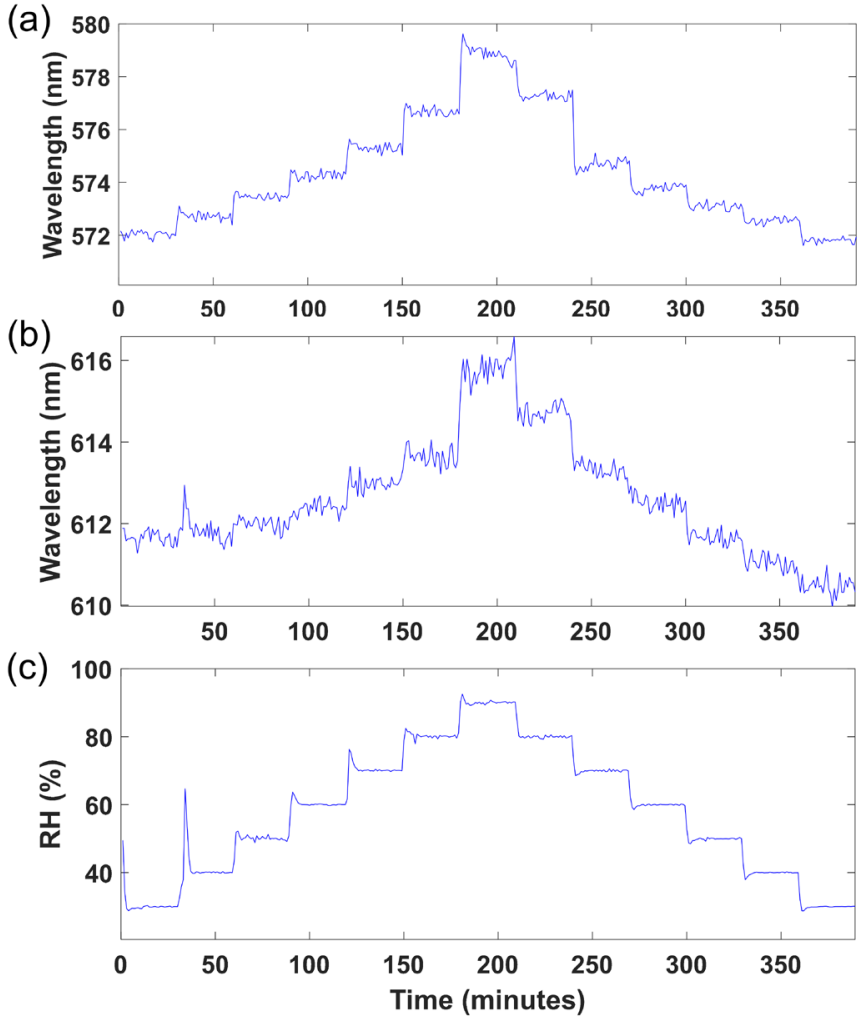

Fig. 9. Wavelength shift of the LMR based sensor when the RH varies from 30 to $90 \%$ in steps of $10 \%$ : (a) coverslip; (b) slide. The relative humidity measured by the electronic sensor is shown in (c).

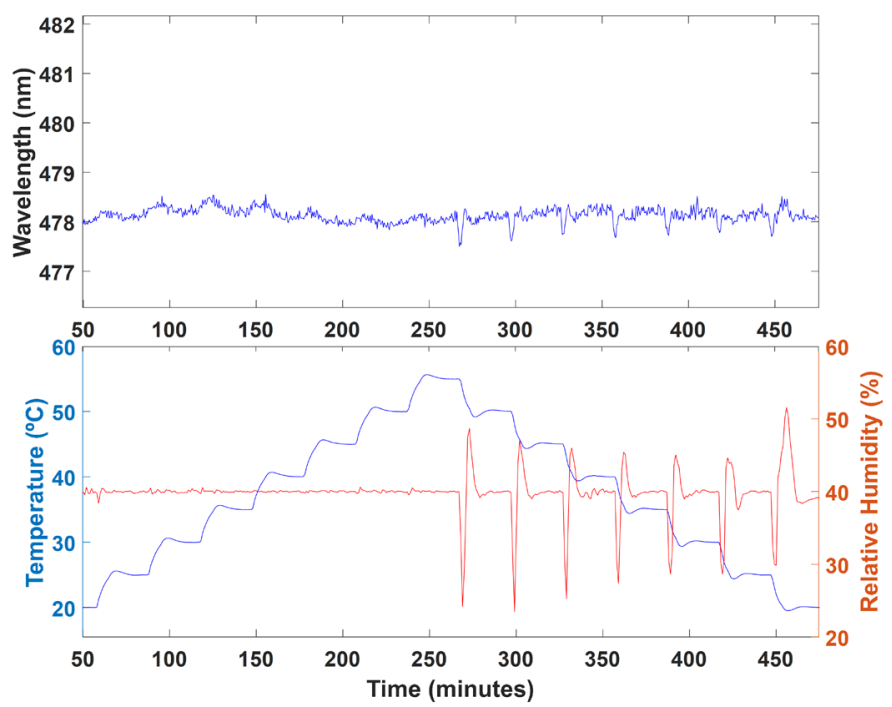

Fig. 10. Temperature cross-sensitivity in an ITO coated glass slide: (a) LMR central wavelength versus (b) temperature and humidity.

Finally, in Fig. 11 the hysteresis of the sensors is analyzed. The maximum variation observed is $0.7 \mathrm{~nm}$ in both devices. This hysteresis is attributed to the properties of the material used for the thin-film, in this case ITO [25]. 

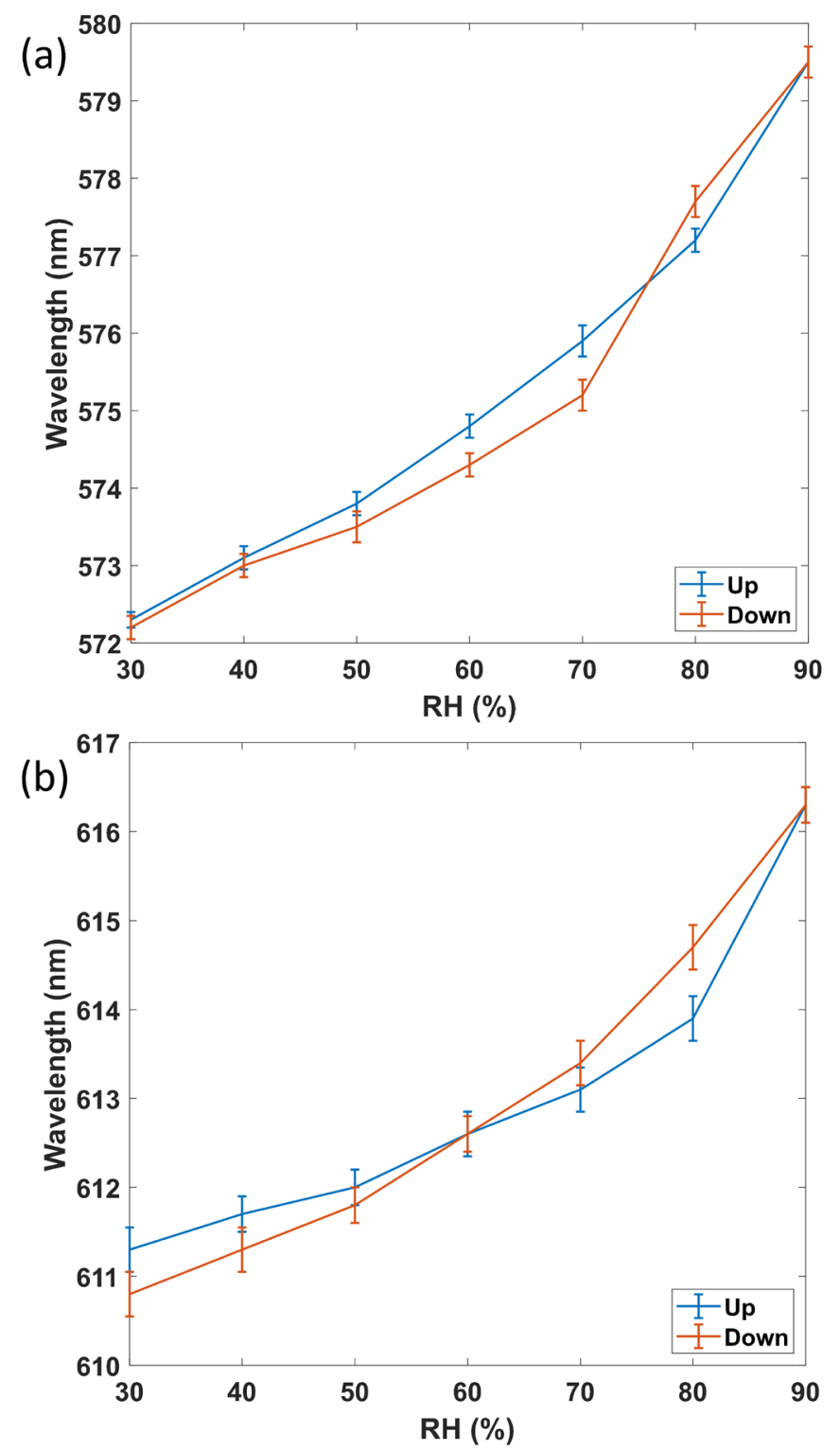

Fig. 11. Variation of the central wavelength of the LMR with the RH: (a) coverslip; (b) glass slide.

\section{CONCLUSIONS}

In this work, a new type of LMR sensor platform based on lateral incidence of light on planar waveguides is shown. This avoids using optical fibre, which is more brittle and requires splices, or the Kretschmann configuration, where it is difficult to couple light because LMRs are typically excited at angles near grazing incidence (near $90^{\circ}$ with the normal of the surface where the thin-film is deposited).

Very simple waveguides have been used for the generation of the LMR and the wavelength range under analysis is located in the visible region, which allows a major reduction of costs. As an example of the utilization of this setup, sensors based on the deposition of indium tin oxide (ITO) have been developed and the devices have been successfully tested as humidity sensors. The behaviour of both the coverslip and the glass slide was similar, though the coverslip presents better characteristics in terms of peak amplitude and noise.
The system described here has enormous industrial and research potential. It joins the good properties of LMR based optical fiber sensors (i.e. an extremely high sensitivity by using materials with a high refractive index and the possibility to tune the resonance at any position in the optical spectrum), with the simplicity and robustness of a setup based on structures as simple as glass slides, which allows its use in real applications to be generalized, as occurred in the case of SPR more than a couple of decades ago. Moreover, like the Dshaped fiber, the non-cylindrically symmetric geometry should allow separating the TE and TM resonances, which could be exploited for obtaining narrower resonances with the advantage that with a planar waveguide the polarizing system should be much easier than with an optical fiber, where an inline polarizer and a polarization controller is needed.

In addition to this, with the setup presented here it is possible to monitor the generation of the LMRs during the deposition process, which could be used for monitoring the thin-film thickness if the refractive index of the material is known. Oppositely, without knowing the refractive index a more complex processing would be needed that considers the wavelength separation between the TE and the TM polarization. In addition to this, the waveguide can be deposited on both sides, which can be used for obtaining a double parameter sensor based on two different resonances or a dual channel microfluidic system could be implemented. Furthermore, the complexity of monitoring the deposition on both sides would increase, but again with the aid of a processing system a new challenge could be to monitor the parallel o serial deposition of two materials on the waveguide. Not to mention the wide range of possibilities in terms of nanostructuring both sides of the waveguide with strips, gratings, nanowires, etc. There is an almost unlimited number of applications and designs that can be developed with this structure

\section{REFERENCES}

[1] Nylander, C. Liedberg, B. Lind, T. "Gas detection by means of surface plasmon resonance," Sensors and Actuators vol. 3, pp. 79-88, 1982.

[2] Kretschmann, E. Raether, H. "Radiative decay of nonradiative surface plasmons excited by light," Zeitschrift für Naturforsch. A vol. 23, pp. 2135-2136, 1968.

[3] I. Del Villar, F. J. Arregui, C. R. Zamarreño, J. M. Corres, C. Bariáin, J. Goicoechea, C. Elosúa, M. Hernáez, P. J. Rivero, A. B. Socorro, A. Urrutia, P. Sánchez, P. Zubiate, D. López, N. De Acha, J. Ascorbe, I. R. Matías, "Optical sensors based on lossy mode resonances," Sens. Act. B Chem., vol. 240, pp. 174-185, 2017.

[4] S. P. Usha, A. M. Shrivastav, B. D. Gupta, "Semiconductor metal oxide/polymer based fiber optic lossy mode resonance sensors: A contemporary study," Optical Fiber Technology vol. 45, pp. 146-166, 2018.

[5] Del Villar, I., Torres, V. \& Beruete, M. "Experimental demonstration of lossy mode and surface plasmon resonance generation with Kretschmann configuration," Opt. Lett. vol. 40, pp. 4739-4382, 2015.

[6] Yang, F. \& Sambles, J. R. "Determination of the optical permittivity and thickness of absorbing films using long range modes," J. Mod. Opt. vol. 44, pp. 1155-1164, 1997.

[7] V. Torres, M. Beruete, P. Sánchez and I. Del Villar "ITO refractometer in the visible and near infrared via lossy mode and surface plasmon 
resonances with Kretschmann configuration," Appl. Phys. Lett. vol. 108, 043507, 2016.

[8] I. Del Villar, M. Hernáez, C. R. Zamarreño, P. Sanchez, C. FernandezValdivielso, F. J. Arregui and I. R. Matías, "Design Rules for Lossy Mode Resonance Based Sensors," Appl. Opt., vol. 51, no. 19, pp. 42984307, 2012.

[9] C. Rhodes, S. Franzen, J. P. Maria, M. Losego, D. N. Leonard, B. Laughlin, G. Duscher, and S. Weibel, "Surface plasmon resonance in conducting metal oxide," J. Appl. Phys., 100, 54905, 2006.

[10] I. Del Villar, C. R. Zamarreño, M. Hernáez, F. J. Arregui and I. R. Matías, "Lossy mode resonance generation with Indium Tin Oxide Coated Optical Fibers for sensing applications," J. Lightwave Technol. vol. 28, no. 1, pp. 111-117, 2010.

[11] S. P. Usha, S. K. Mishra, B. D. Gupta, "Fiber optic hydrogen sulfide gas sensors utilizing $\mathrm{ZnO}$ thin film/ $\mathrm{ZnO}$ nanoparticles: A comparison of surface plasmon resonance and lossy mode resonance," Sens. Act. B Chem.. 218, 196-204, 2015.

[12] Mateusz Smietana, Michał Sobaszek, Bartosz Michalak, Paweł Niedziałkowski, Wioleta Białobrzeska, Marcin Koba, Petr Sezemsky, Vitezslav Stranak, Jakub Karczewski, Tadeusz Ossowski, and Robert Bogdanowicz, "Optical Monitoring of Electrochemical Processes With ITO-Based Lossy-Mode Resonance Optical Fiber Sensor Applied as an Electrode," J. Lightwave Technol., vol. 36 (4), pp. 954-960, 2018.

[13] Divya Tiwari, Kevin Mullaney, Serhiy Korposh, Stephen W. James, Seung-Woo Lee, Ralph P. Tatam, "An ammonia sensor based on Lossy Mode Resonances on a tapered optical fibre coated with porphyrinincorporated titanium dioxide," Sens. Act. B Chem., vol. 242, pp. 645652, 2017.

[14] A. Ozcariz, C. R. Zamarreño, P. Zubiate, and F. J. Arregui, "Is there a frontier in sensitivity with Lossy mode resonance (LMR) based refractometers?" Sci. Rep., vol. 7(1), 10280, 2017.

[15] F. Chiavaioli, P Zubiate, I. Del Villar, C.R Zamarreño, A Giannetti, S. Tombelli, C. Trono, F.J. Arregui, I.R. Matias, F. Baldini, "Femtomolar Detection by Nanocoated Fiber Label-Free Biosensors," ACS Sensors, vol. 3, pp. 936-943, 2018.

[16] Hamid Farahani, Rahman Wagiran and Mohd N. Hamidon, "Humidity sensors principle, mechanism, and fabrication technologies: A comprehensive review," Sensors, vol. 14, pp. 7881-7939, 2014.

[17] J. Ascorbe, J. M. Corres, F. J. Arregui and I. R. Matias, "Recent Developments in Fiber Optics Humidity Sensors," Sensors, vol. 17 (4), pp. 893, 2017.

[18] L. Alwis, T. Sun, K. T. V. Grattan, “Optical fibre-based sensor technology for humidity and moisture measurement: Review of recent progress," Measurement, vol. 46, pp. 4052-4074, 2013.

[19] M. Rubin. "Optical properties of soda lime silica glasses," Sol. Energy Mater., vol. 12, pp. 275-288, 1985.

[20] N. Sultanova, S. Kasarova and I. Nikolov. "Dispersion properties of optical polymers," Acta Physica Polonica A, vol. 116, pp. 585-587, 2009.

[21] H. Kim, J. S. Horwitz, G. Kushto, A. Piqué, Z. H. Kafafi, C. M. Gilmore, and D. B. Chrisey, "Effect of film thickness on the properties of indium tin oxide thin films," J. Appl. Phys., vol. 88, 6021, 2000.

[22] A. B. Socorro, I. Del Villar, J. M. Corres, F. J. Arregui and I. R. Matias, "Spectral width reduction in lossy mode resonance-based sensors by means of tapered optical fibre structures," Sens. Act. B Chem., vol. 200, pp. 53-60, 2014.

[23] C. Guillén, J. Herrero. "Stability of sputtered ITO thin films to the dampheat test," Surface \& Coatings Technology, vol. 201, pp. 309-312, 2006.

[24] P. Sanchez, C. R. Zamarreño, M. Hernaez, I. Del Villar, C. FernandezValdivielso, I. R. Matias and F. J. Arregui, "Lossy mode resonances toward the fabrication of optical fiber humidity sensors," Meas. Sci. Technol., vol. 23014002 (6pp), 2012.

[25] M. Hernaez, C.R. Zamarreño, I. Del Villar, F.J. Arregui, I.R. Matias, "Optical fiber humidity sensor based on lossy mode resonances," International Journal on Smart Sensing and Intelligent Systems, vol. 2(4), pp. 653-660, 2009.

Omar Fuentes is an Automatic Engineer (1996) from Higher Polytechnic Institute "José A. Echeverria (CUJAE), La Habana, Cuba". Currently he works as Assistant Professor in the Department of Telecommunication and Electronic Engineering in Pinar del Río University (UPR).
Jesus M. Corres received the MS degree in electrical engineering from the Public University of Navarra, Pamplona, Spain, in 1996 and the PhD degree from the Public University of Navarra, Pamplona, Spain in 2003. Currently he works as Associate Professor in the Department of Electrical and

Electronic Engineering (UPNA). His main research is the development of fiber optic sensors using nanostructured materials for biomedical, environmental and safety applications. He is the author or co-author of more than 100 publications and serves as associate editor of IEEE Sensor Letters and Hindawi Journal of Sensors.

Ignacio R. Matias received the M.S. degree in Electrical and Electronic Engineering and his Ph.D. degree in Optical Fiber Sensors from the Polytechnic University of Madrid (UPM), Madrid, Spain, in 1992 and 1996, respectively. He became a Lecturer at the Public University of Navarra (Pamplona, Spain) in 1996, where presently he is a Permanent Professor. He has co-authored more than 300 chapter books, journals and conference papers related to optical fiber sensors and passive optical devices and systems. He is a Senior Editor of "IEEE Sensors Journal". He is an IEEE Senior member. He is a Topical Editor of the IEEE Sensors Journal

Ignacio Del Villar received his MS degree in Electrical and Electronic Engineering and his Ph.D. degree, specialty in Optical Fiber Sensors, in 2002 and 2006, respectively, from the Public University of Navarra (UPNA). During 2004 was a visiting scientist at the Institute d'Optique (Orsay, France) and in 2005 he was a visiting scientist at the Applied Physics Department of the University of Valencia (Burjassot, Spain). $\mathrm{He}$ is presently a Reader at the Public University of Navarra since 2008, an Associate Editor of the Optics \& Laser Technology Journal since 2012 and of Sensors Journal since 2017. His research interest includes optical fiber sensors and the effect of nanostructured coatings deposited on waveguides, where he has co-authored more than 100 chapter books, journals and conference papers 